\title{
SEM/EDS Evidence that Cylindrical Structures Emanating from Dentinal Tubules are Involved in Mediating Calcium Phosphate Formation.
}

\author{
B. Leite ${ }^{1}$, D. Guarrera ${ }^{2}$ R.-C, Hsia $^{3} \&$ G. Hack ${ }^{3}$ \\ ${ }^{1}$ Thermo Fisher Scientific, 2552 Verona Road, Madison, WI, 53711 \\ ${ }^{2}$ JEOL USA, Inc., Peabody, MA, 01960 \\ ${ }^{3}$ University of Maryland Dental School, Baltimore, MD, 21201
}

Dentinal hypersensitivity is an important topic of modern dental research. We have identified cylindrical structures emanating from dentinal tubules that may be associated with dentinal hypersensitivity. We have studied in detail a human molar tooth found to be exhibiting thousands of such cylinders. Our working hypothesis is that these cylinders aid in the movement of dentinal fluids, which generates dentinal pain or tooth sensitivity. Physiologically speaking, it is crucial to maintain this status as a warning to the individual's health as a whole, similarly to the sensation of hot and cold, which keep us away from harmful situations. We have used a combination of Scanning Electron Microscopy (SEM) and Energy Dispersive Spectroscopy (EDS) [1] to evaluate this problem.

Our first objective was to study morphological details of these cylindrical structures. We measured approximately the diameter of 200 dentinal tubules. In the area analyzed, the diameter varied considerably, ranging from completely closed to 1 micron (Figs 1A \& 1B). We also noticed that approximately 58\% were opened and $42 \%$ were closed. In addition, we have observed a limited number of large sized openings, which represent less than $5 \%$ of the total. Since this is preliminary work, we cannot access the meaning of these findings in relation to the physiological status of the dentine area examined, except for possibly the overall hypersensitive condition. These dentinal tubules progress from completely open to totally occluded. In some areas, groups of tubules exhibit complete occlusion by amorphous material (AM) (Fig 1A).

We compared the elemental composition of the dentine matrix itself with the tubular regions (Fig 2). We were initially searching for differences in $\mathrm{Ca}, \mathrm{P}$ and $\mathrm{O}$. However, observation of these areas consistently showed evidence of different levels of $\mathrm{Na}, \mathrm{Mg}, \mathrm{Cl}$ and $\mathrm{Si}$, which were only detected in the tubular areas. Locally adjusting the concentration of $\mathrm{Na}$ and $\mathrm{Mg}$ in the tubular areas is consistent with higher fluidity, as these elements are common electrolytes [2]. The combined action of $\mathrm{Na}, \mathrm{Mg}$, $\mathrm{Cl}$ and $\mathrm{Si}$ can account for more flow within these opened channels. In a recent publication [3], the role of these structures have been pointed out as key structures in avoiding mineralization by precluding $\mathrm{Ca}$ and $\mathrm{P}$ from forming calcium phosphate (hydroxyapatite) crystals. In conclusion, we speculate that these channels are kept highly demineralized contrasting to the highly mineralized dentine matrix to allow continued communication.

References

[ 1 ] Thermo Scientific, Noran System Seven. Spectral Imaging tools.

[ 2 ] Ehrlich et al, 2008. Micron 39: 1062-1091.

[ 3 ] Hack et al, 2010. AADR 2010, Washington, DC, USA. 

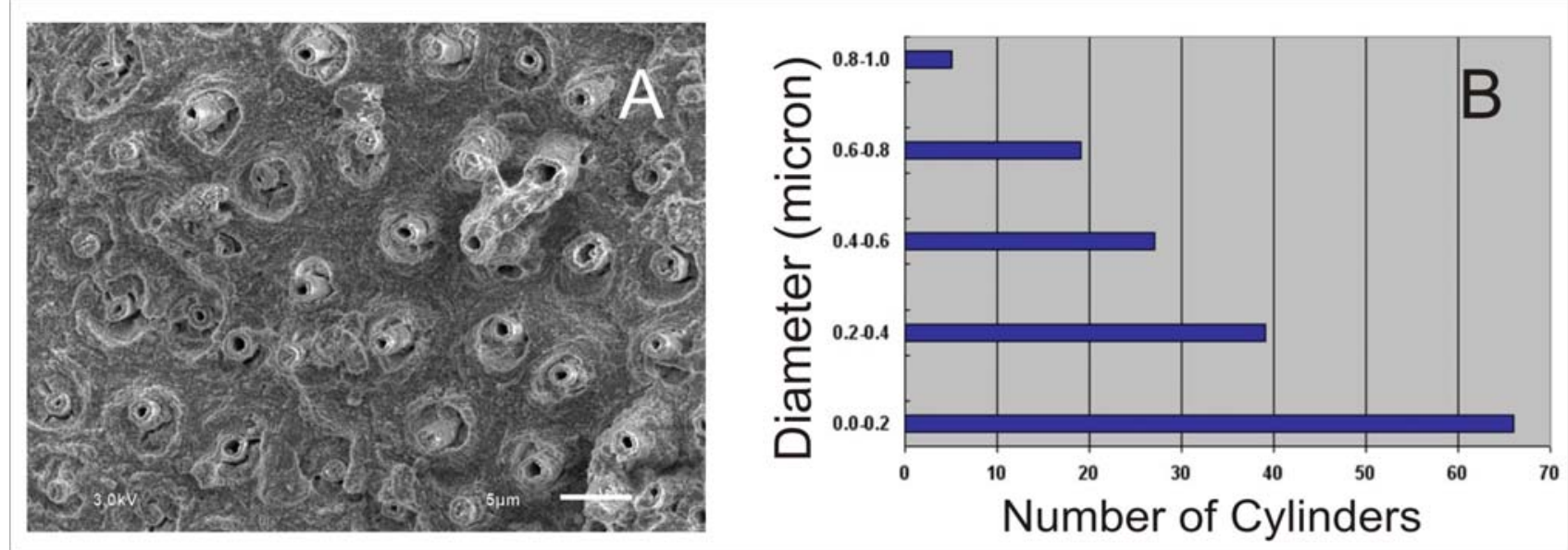

Fig 1 - General view of a hypersensitive dentine area exhibiting cylindrical structures (known as "tooth worms") emanating from hypersensitive dentine openings (A). Notice that most of these openings have narrow diameters (B).

\begin{tabular}{|l|c|c|c|c|}
\hline \multicolumn{5}{|c|}{ Comparative Table of Typical Dentine Matrix and Opening Areas } \\
\hline Elements & Opening W\% & W\% error & Matrix W\% & W\% error \\
\hline C K & 9.17 & $+/-0.19$ & 10.5 & $+/-0.57$ \\
\hline O K & 29.85 & $+/-0.26$ & 35.56 & $+/-0.27$ \\
\hline Na K & $\mathbf{0 . 8 7}$ & $+/-0.10$ & $\mathbf{1 . 0 3}$ & $+/-0.09$ \\
\hline Mg K & $\mathbf{0 . 5 9}$ & $+/-0.07$ & $\mathbf{0 . 2 7}$ & $+/-0.04$ \\
\hline Si K & $\mathbf{0 . 2 1}$ & $+/-0.05$ & $\mathbf{0}$ & 0 \\
\hline P K & 20.51 & $+/-0.20$ & 17.9 & $+/-0.18$ \\
\hline Cl K & $\mathbf{0 . 5 3}$ & $+/-0.08$ & $\mathbf{0 . 3 7}$ & $+/-0.07$ \\
\hline Ca K & 38.26 & $+/-0.35$ & 34.36 & $+/-0.31$ \\
\hline
\end{tabular}
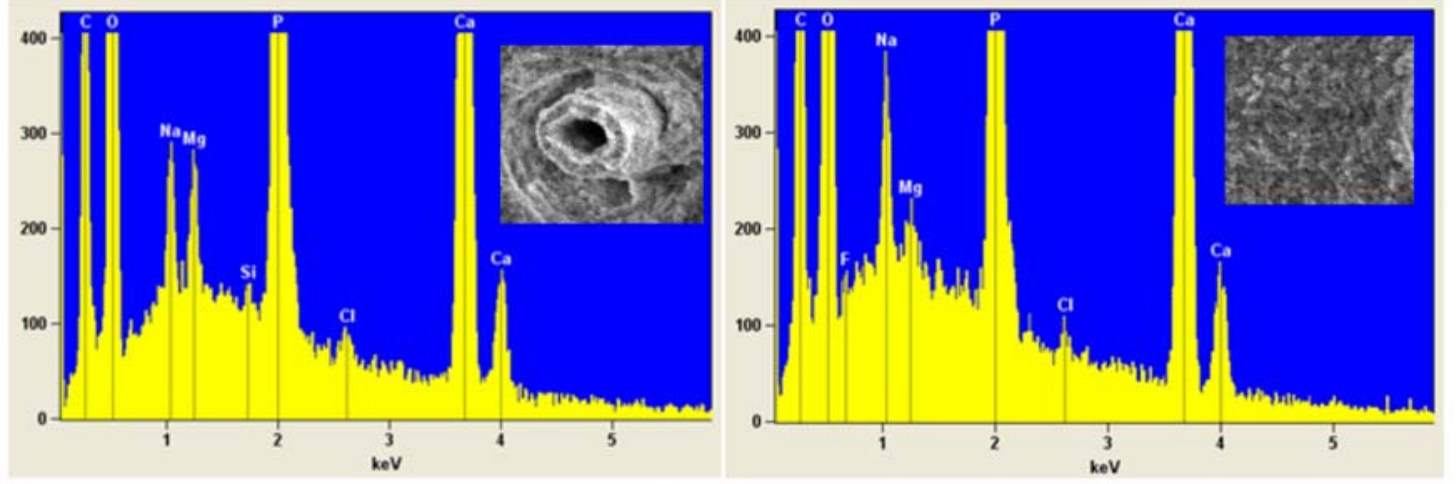

Fig 2 - The table is comparing element profiles found in areas where openings (left image) and dentine matrix (right image) are located. The table is accompanied by the representative spectra these areas, highlighting the differences on the low concentration elements. This was preferred due to the intense calcium and phosphorous background. 